\title{
Abuso de drogas e suas consequências na saúde bucal: uma revisão de literatura
}

\section{Drug Abuse and its Consequences in Oral Health: A Review of Literature}

\begin{abstract}
Lidia Audrey Rocha Valadas Marques Cirurgiã-dentista. Especialista em Farmacologia Clínica. Doutoranda do Programa de Pós-graduação em Inovação e Tecnologia em Desenvolvimento de Medicamentos. Universidade Federal do Ceará, Fortaleza, CE, Brasil.
\end{abstract}

Mara Assef Leitão Lotif Cirurgiã-dentista. Especialista em Farmacologia Clínica. Mestranda do Programa de Pós-graduação em Odontologia. Universidade Federal do Ceará, Fortaleza, CE, Brasil.

Edilson Martins Rodrigues Neto Farmacêutico. Especialista em Farmacologia Clínica, Toxicologia e Assistência Farmacêutica. Mestre em Farmacologia. Doutorando do Programa de Pós-graduação em Farmacologia. Universidade Federal do Ceará, Fortaleza, CE, Brasil.

Antônio Pergentino Nunes Neto Graduação em Odontologia. Universidade Federal do Ceará, Fortaleza, CE, Brasil.

Cândida Carolinne Santos Alves Melo Graduação em Odontologia. Universidade Federal do Ceará, Fortaleza, CE, Brasil.

PATRÍcIa LeAL DANTAS Lobo Cirurgiã-dentista. Especialista em Odontopediatria e Ortodontia. Mestre em Odontologia (Odontopediatria, Doutora em Farmacologia. Professora das disciplinas de Odontopediatria e Ortodontia. Universidade Federal do Ceará, Fortaleza, CE, Brasil.

\begin{abstract}
RESUMO
Introdução: O abuso de drogas é um dos principais problemas sociais e de saúde pública do mundo. Alguns estudos sugerem um aumento significativo do número de dependentes químicos com manifestações orais decorrentes do consumo de drogas, bem como a falta de conhecimento por parte dos cirurgiões-dentistas quanto ao assunto. Objetivos: Objetivou-se neste artigo realizar um levantamento na literatura acerca das principais consequências na cavidade bucal decorrentes do consumo das drogas lícitas e ilícitas mais utilizadas. Metodologia: Para isso, realizou-se uma revisão de literatura de artigos publicados a respeito do assunto, sobretudo por meio dos bancos de dados Scopus, Pubmed e LILACS, utilizando como descritores as seguintes palavras: "manifestações bucais", "drogas de abuso", "alterações bucais". Dessa forma, foram selecionados 28 artigos publicados entre 1985 e 2012, além de livros sobre o assunto. Conclusão: O uso de substâncias psicoativas pode gerar vários danos à saúde oral, que vão desde a halitose e gengivite ao câncer de boca. Com o cirurgião dentista realizando o reconhecimento inicial, o dependente químico pode receber tanto o tratamento odontológico quanto ser encaminhado aos demais profissionais da saúde, visando o acompanhamento multidisciplinar de acordo com as necessidades individuais de cada paciente.

Palavras-chave: Manifestações bucais; Drogas ilícitas; Diagnóstico.
\end{abstract}

\begin{abstract}
Introduction: Drug abuse is a major social problems and public health worldwide. Some studies suggest a significant increase in the number of addicts with oral manifestations resulting from drug use, and lack of knowledge on the part of dentists for that matter. Objective: The objective of this article is to survey the literature on the major consequences in the oral cavity arising from the consumption of illicit and licit drugs and more used. Methodology: For this, it conducted a literature review of articles published primarily through Scopus, Pubmed and LILACS, using as descriptors the following words: "utterances", "drugs of abuse", "oral
\end{abstract}


abnormalities". Thus, we selected 28 articles published between 1985 and 2012, as well as books on the subject. Conclusion: The use of psychoactive substances can cause severe damage to oral health ranging from halitosis and gingivitis to mouth cancer. With the dentist performing the initial recognition, the addict can receive dental treatment as well be referred to other health professionals, aimed at multidisciplinary according to the individual needs of each patient.

Keywords: Oral manifestations; Street drugs; Diagnosis.

\section{INTRODUÇÃO}

O abuso de drogas é um dos principais problemas sociais e de saúde pública do mundo. Entretanto, as drogas envolvem várias questões que vão além da saúde, por exemplo, o tráfico, violência e aspectos morais. ${ }^{1-2}$

Os efeitos do uso abusivo de álcool e outras drogas são grandes determinantes para a morbimortalidade, nem sempre percebidos de modo claro pela população, pois os usuários geralmente apresentam atitudes contraditórias e existe o preconceito a respeito deles. Tal preconceito pode ser encontrado em diversas culturas, variando em intensidade de acordo com certos fatores (tipo de droga, sexo e idade do usuário, classe social) e com o período histórico. Ainda nesse contexto, podese abordar a questão das drogas tidas como "menos nocivas", desse modo, sendo mais toleradas pela sociedade. ${ }^{3}$

Alguns estudos sugerem um aumento significativo do número de dependentes químicos com manifestações bucais decorrentes do consumo de drogas, bem como a falta de conhecimento por parte dos cirurgiõesdentistas quanto ao assunto. Percebe-se que é preciso uma atenção especial do cirurgiãodentista durante 0 atendimento desses pacientes, pois em muitos casos serão os profissionais dessa área os primeiros a terem a oportunidade de diagnosticar o surgimento de possíveis alterações ocorridas devido ao consumo de cigarro, álcool ou drogas ilícitas. ${ }^{4,5}$

Visto o desconhecimento e a escassez de informações na literatura quanto ao assunto, objetivou-se neste artigo realizar um levantamento na literatura acerca das principais consequências na cavidade bucal decorrentes do consumo das drogas lícitas e ilícitas mais utilizadas.

\section{MATERIAIS E MÉtodos}

Neste estudo, foi realizada uma revisão de literatura sobre as manifestações bucais decorrentes do consumo de drogas de abuso, problemática ainda pouco abordada na literatura, na qual discorremos sobre a classificação dessas substâncias, prevalência de uso e principais manifestações bucais observadas. Para isso, foi realizada uma revisão de literatura de artigos publicados acerca das principais drogas consumidas, sobretudo por meio dos bancos de dados LILACS, SCOPUS e PUBMED, utilizando como descritores as seguintes palavras: "manifestações orais", "drogas de abuso", "alterações bucais" e seus respectivos termos em inglês. Foram obtidos um total de 46 artigos e selecionados 28, além de referências como monografias e livros. Como critérios de inclusão, foram selecionados artigos publicados nos idiomas inglês e português, no período de 1985 a 2012, na forma de artigos epidemiológicos e relatos de caso clínico.

\section{REVISÃo de LITERATURA}

Álcool
O álcool é a droga mais consumida e aceita
socialmente no mundo. Segundoa Organização
Mundial de Saúde, aproximadamente dois
bilhões de pessoas no mundo consomem
bebidas alcoólicas. O uso indevido do álcool
é o fator principal para ele ser considerado


o maior problema de saúde pública mundial, sendo um dos principais fatores que contribuem diretamente para a diminuição da qualidade da saúde pública no planeta, principalmente na América Latina, onde o uso é cerca de quatro vezes maior do que a média mundial. ${ }^{1}$

O uso abusivo do álcool implica efeitos deletérios tanto para a saúde sistêmica, quanto para a saúde bucal. Em relação à saúde bucal, o alto consumo do etanol, por apresentar natureza irritante da mucosa oral, está relacionado ao aumento do risco para doenças periodontais e cáries. Somado a esses efeitos causados pelo álcool, os dependentes normalmente apresentam negligência da higiene oral, favorecendo a colonização de microrganismos formadores da placa bacteriana. ${ }^{5-6}$

As principais alterações periodontais relacionadas ao abuso do álcool são gengivite úlceronecrosante, periodontite, recessão gengival, além de maior quantidade de perda do osso alveolar e diminuição do fluxo salivar. ${ }^{7}$

O fluxo salivar normal é um importante fator de proteção contra o surgimento de lesões de cárie dentária e doenças periodontais. Quando o fluxo salivar se encontra reduzido, lesões cariosas podem acometer os dentes mais facilmente, principalmente pela redução da capacidade tampão da saliva e consequente incapacidade de remoção mecânica do biofilme, que está aderido à superfície do dente. Além disso, a gengiva desses pacientes se encontra em um constante processo inflamatório, causando sangramento e formação de bolsas periodontais. ${ }^{8}$

Alguns estudos epidemiológicos apontam o consumo de álcool como um fator de risco para o desenvolvimento do carcinoma espinocelular, que é o câncer de boca mais comum. Entretanto, o consumo de álcool atua de forma indireta na formação de neoplasias malignas, pois não possui a capacidade de transformar uma célula normal em maligna, mas altera o epitélio da mucosa e das glândulas, portanto, é considerado um coadjuvante na carcinogênese oral. ${ }^{9}$

Devido a todas essas predisposições ao surgimento de doenças bucais, os usuários de álcool devem receber uma atenção especial dos cirurgiões-dentistas, principalmente no que se refere ao uso de medicamentos para controle da dor e de infecções. Atenção especial deve ser dada à associação do paracetamol com álcool, devido ao potencial hepatotóxico dessa associação. ${ }^{10}$

\section{Anfetaminas}

A anfetamina é classificada como uma droga sintética, ou seja, fabricada em laboratório e que tem o poder de estimular o sistema nervoso central, fazendo que o cérebro trabalhe muito e mais rápido que o normal. Isso possibilita que os usuários fiquem hiperativos e sem sono, apresentando efeitos semelhantes aos da cocaína. ${ }^{11}$

Entre as principais manifestações bucais associadas ao uso de anfetaminas encontrase o fluxo salivar reduzido, xerostomia, doença periodontal, cáries rampantes (cáries que levam a um rápido processo de destruição coronária) e bruxismo, que causam desgaste anormal dos dentes. ${ }^{12}$

O tratamento odontológico de usuários de anfetaminas é bastante complexo, exigindo do cirurgião-dentista uma anamnese e um exame clínico minuciosos para se elaborar qualquer plano de tratamento, visto que os efeitos do consumo de metanfetaminas são evidentes em vários órgãos, principalmente ao nível dos sistemas cardiovascular e nervoso central. Além disso, a cooperação do paciente é essencial, principalmente se ele parar de usar a droga, já que ela age de maneira rápida e destruidora. ${ }^{13}$

\section{Maconha}

A maconha é uma erva de origem da Ásia Central, cujo nome científico é Cannabis 
sativa. Suas propriedades psicoativas estão relacionadas ao clima, altitude e solo, onde a mesma requer clima quente e seco e umidade adequada do solo. A maconha, depois do álcool, é a droga mais consumida no mundo, tendo como constituinte químico majoritário, responsável pelos efeitos no SNC, o $\Delta \square$-Tetraidrocanabinol. Essa substância estabelece no organismo um efeito supressor da resposta imune, tanto a nível humoral quanto celular. ${ }^{14,15,16}$

Os usuários de maconha, geralmente, apresentam uma pior saúde bucal comparado aos não usuários. Entre as principais manifestações bucais provocadas pelo uso da maconha encontram-se a cárie dentária, doenças periodontais, estomatite canábica, xerostomia e candidose. Assim como o cigarro, a fumaça da maconha possui um potencial cancerígeno, ou seja, ela tem a capacidade de alterar e lesionar o epitélio da mucosa oral, sendo frequente o surgimento de leucoplasia e eritroplasia, principalmente quando o usuário também faz uso do cigarro. ${ }^{16}$

Além disso, a maconha tem a capacidade de provocar efeitos no sistema nervoso parassimpático que, em associação ao uso dos anestésicos locais com vasoconstrictores utilizados na clínica odontológica, pode induzir à taquicardia no paciente. ${ }^{17}$

\section{Cocaína}

A cocaína é uma droga estimulante do sistema nervoso central, extraída das folhas da planta Erythroxylon coca. Essa droga pode ser consumida sob a forma de cloridrato de cocaína, um sal hidrossolúvel, que normalmente é aspirado por via nasal. Existem também as apresentações alcalinas, que são voláteis a baixas temperaturas e podem ser fumadas em forma de "cachimbos", como é o caso do crack, um derivado da cocaína. ${ }^{18}$

Quando a cocaína é aspirada, seus efeitos vasoconstritores fazem que o uso prolongado resulte em necrose e perfuração do septo nasal. Esse efeito vasoconstrictor produz irritação da mucosa do palato, que pode evoluir para perfuração nasal e destruição dos ossos maxilares. Provavelmente isso é facilitado pela forma de consumo, que é mais frequente por aspiração. ${ }^{19}$

Entre os principais efeitos da cocaína e seus derivados, como o crack ou óxi, encontram-se a gengivite ulceronecrosante, periodontite avançada, laceração gengival e principalmente lesões na gengiva semelhantes a queimaduras, alto índice de cáries e perdas dentárias, candidose e bruxismo. Esses efeitos se devem principalmente a alguns contaminantes do produto final, que são substâncias corrosivas/irritantes, como o ácido clorídrico e a gasolina. ${ }^{16,20,21}$

A cocaína é a droga ilícita mais frequentemente associada a óbitos, induz a liberação de norepinefrina e, por conseguinte, bloqueia a sua recaptação pelas terminações nervosas adrenérgicas, assim interage com as aminas simpatomiméticas contidas nas soluções anestésicas, potencializando efeitos de vasoconstrição, aumento da pressão arterial e taquicardia. Portanto, usuários de cocaína são pacientes de risco para toda e qualquer complicação cardiovascular. Além disso, essa associação leva a uma constrição no baço, o que gera a produção exacerbada de eritrócitos, aumentando a viscosidade sanguínea, que pode levar à formação de trombos nos vasos sanguíneos. Assim, a administração de anestesia local deve ser feita em doses mínimas, pois não há dosagem segura para um paciente usuário de cocaína. ${ }^{20,21}$

Ecstasy
O ecstasy possui como principal
constituinte em seus comprimidos o
3,4-metilenodioximetanfetamina (MDMA), que
é um derivado da anfetamina. Seus efeitos
variam de acordo com a dose, frequência e
duração do uso. ${ }^{22}$


Entre as principais consequências na cavidade bucal decorrentes do consumo do ecstasy podemos encontrar a xerostomia, cáries dentárias (associado ao consumo excessivo de doces na tentativa do usuário conter o bruxismo), bruxismo, sensibilidade dentinária, periodontites, parestesia de nervos faciais, úlceras e edemas. Usuários relatam também tremores faciais que causam mordeduras involuntárias dos lábios e da língua. ${ }^{16,21}$

\section{Nicotina}

A nicotina, substância presente na Nicotianatabacum, é uma droga originária da América do Sul, sendo umas das drogas mais antigas e a mais usada atualmente, sob forma de cigarro. Além disso, é considerada a maior causa de morte evitável no mundo. ${ }^{23}$

A nicotina age diretamente, diminuindo a resposta imunológica dos usuários, deixandoos propensos a doenças sistêmicas e bucais. Entre as principais manifestações orais relacionadas ao uso da nicotina se encontram a gengivite, a periodontite com formação de bolsas periodontais, cáries, perdas dentárias e halitose. Somado a isso está o grande risco de desenvolvimento de lesões com potencial maligno, ou seja, lesões que podem predispor ao câncer bucal, como a leucoplasia, que é a lesão que apresenta o maior potencial de transformação maligna, o leucoedema, que não é necessariamente uma lesão, mas que se apresenta de forma mais acentuada em fumantes do que em não fumantes e pode ser confundido com a leucoplasia. Todas as manifestações causadas pela nicotina possuem um risco aumentado quando existe uma combinação do tabagismo com o consumo excessivo de álcool, principalmente o câncer bucal. ${ }^{21,24,25}$

Dentistas têm contato frequente com seus pacientes e também podem explorar em suas consultas os efeitos nocivos do tabaco na cavidade bucal, como manchamento de dentes, halitose, doença periodontal e perda dentária. Desse modo podem ter fundamental importancia na cessação do tabagismo de seus pacientes, ao informar sobre os malefícios desse hábito. ${ }^{26}$

\section{Dıscussão}

O aumento do consumo de drogas no mundo, em especial nos países capitalistas, é um fato diretamente relacionado à oferta de novas drogas, que surgem a cada dia, e ao lucro gerado por elas. O álcool e a nicotina, por serem as drogas mais utilizadas e consideradas lícitas, têm o seu uso estimulado pela sociedade capitalista, enquanto as drogas ilícitas são controladas pelo narcotráfico. A dependência de todas essas drogas causa proporções epidêmicas, que influenciam tanto na saúde quanto no aspecto social. ${ }^{27}$

Tratando-se do consumo global de drogas ilícitas, a maconha atualmente é a mais utilizada, seguida das anfetaminas e ecstasy. Depois dessas, encontram-se a cocaína e os seus derivados, que vêm ganhando destaque, devido ao aumento e às consequências que a cocaína acarreta na saúde e na vida social dos usuários. ${ }^{28}$

Toda substância química de abuso tem um poder de causar danos no tecido bucal, mesmo tendo a sua classificação como lícita ou ilícita. A utilização contínua de substâncias tóxicas com potencial dependência tem a capacidade, de modo demasiado, de aumentar o nível CPOD (dentes cariados, perdidos, obturados e com extração indicada); além de causar outros danos, como gengivite, halitose, estomatite, bruxismo, desgastes dentais, queilite angular. ${ }^{29}$

Um relatório mundial sobre a saúde revela que o consumo de substâncias psicoativas é responsável, direta e indiretamente, por 8,9\% das taxas de doenças da população. Dados publicados nesse levantamento demonstraram que o cigarro representava $4,1 \%$, o álcool $4 \%$, 
e as drogas ilícitas $0,8 \%$ dos responsáveis, em 2000, por essas doenças. Essa situação alarmante pode ser correlacionada com dependências crescente do consumo de substâncias psicoativas lícitas e ilícitas, resultando em uma gama de problemas sociais e sanitários. ${ }^{30}$

Associado a esses problemas, há uma carência de informações sobre o tema voltado para a odontologia, para subsidiar a conduta dos profissionais frente a esse sério problema de saúde pública, que afeta a qualidade de vida de usuários e familiares. ${ }^{31}$

Ao realizar atendimento odontológico aos usuários de drogas, o cirurgião-dentista deve estar atento para evitar possíveis complicações. É imprescindível um exame clínico com a anamnese detalhada antes de realizar qualquer procedimento, e estar sempre atento ao paciente, apto a reconhecer sinais do uso dessas substâncias que podem não ter sido relatadas durante a anamnese. A grande importância disso é planejar um procedimento seguro, principalmente quando será necessário utilizar anestésicos locais, que são amplamente usados em vários procedimentos odontológicos e apresentam reações importantes no organismo desses pacientes. ${ }^{30,25}$

O cirurgião-dentista pode contribuir de maneira substancial no processo de reabilitação desses pacientes, aliviando a dor e auxiliando na interação social, por meio de uma reabilitação oral plena, visando o processo de recuperação e resgatando o ser humano em todos os aspectos, diminuindo o estigma psicológico das drogas. ${ }^{31}$

\section{Conclusão}

O uso de substâncias psicoativas pode gerar vários danos à saúde bucal, que vão desde a halitose e gengivite ao câncer de boca. Essas drogas, muitas vezes, aumentam o limiar de dor do usuário, mascarando a sinto- matologia dolorosa de lesões orais, agravando a situação, pois o paciente demorará mais a buscar um serviço de saúde.

Com o cirurgião-dentista realizando o reconhecimento inicial, o dependente químico pode receber tanto o tratamento odontológico quanto ser encaminhado aos demais profissionais da saúde, visando o acompanhamento multidisciplinar, de acordo com as necessidades individuais de cada paciente.

\section{REFERÊNCIAS}

1. Laranjeira R., Pinsky I., Zaleski M., Caetano RI. Levantamento Nacional sobre os padrões de consumo de álcool na população brasileira, 1.ed., Brasília: Editora SENAD; 2007.

2. Noto AR, Baptista MC, Faria ST, Nappo AS, Galduróz JCF, Carlini EA. Drogas e saúde na imprensa brasileira: uma análise de artigos publicados em jornais e revistas. Cad. Saúde Pública, 2003; 19(1): 69-79.

3. Luis MAV, Lunetta ACF. Álcool e outras drogas: Levantamento preliminar sobre a pesquisa produzida no Brasil pela enfermagem. Rev Latino-am Enfermagem, 2005; 13(1): 1.229-30.

4. Colodel EV, Silva ELFM, Zielak JC, Zaitter W, Michel-Crosto E, Pizzatto E. Alterações bucais presentes em dependentes químicos. RSBO, 2009; 6(1): 44-8.

5. Fernandes JP, Brandão VSG, Lima AAS. Prevalência de lesões cancerizáveis bucais em indivíduos portadores de alcoolismo. Rev. Bras. Cancerol. 2008; 54(3): 239-44.

6. Péret ACA, Bonato KB. A participação do dentista na equipe multidisciplinar para o tratamento do paciente alcoolista. Arq. Bras. Odontol., 2008; 4(2): 70-5.

7. Faustino SES, Stipp ACM. Effects of chronic alcoholism and alcoholic detoxication on rat submandibular glands. Morphometricstudy. J Appl Oral Sci, 2003; 11(1): 21-6.

8. Pinto-Coelho CM, Souza TCS, Dare AMZ, Pereira CCY, Cardoso. Clínicas da Xerostomia: abordagens sobre o diagnóstico e tratamento. Rev. Odontol. Araç., 2002; 56(4): 295-300. 
9. Llewellyn CD, Johnson NW, Warnakulasuriya KA. Risk factors for oral cancer in newly diagnosed patients aged 45 years and younger: a case-control study in Southern. England. J Oral Pathol Med., 2004; 33(9): 525-32.

10. Cantwell B, McBridge AJ. Self detoxification by anphetamine dependent patients: a pilot study. Drug Alcohol Depend, 1998; 49(1): 157-63.

11. Amaral AS, Guimarães MI. Manifestações orais do uso de metanfetaminas. Rev. Port. Estomatol. Med. Dent. Cir. Maxilofac., 2012; 53(1): 175-80.

12. Hamamoto DT, Rhodus NL. Methamphetamine abuse and dentistry. Oral Dis., 2009; 15(1): 27-37.

13. Coutinho MPL, Ludgleydson FA, Gonties B. Uso da maconha e suas representações sociais: estudo comparativo entre universitários. Psicol. Est., 2004; 9(3): 469-77.

14. Almeida PP, Novaes MAFP, Bressan RA, Lacerda ALT. Revisão: funcionamento executivo e uso de maconha. Rev. Bras Psiquiatr., 2008; 30(1): 69-76.

15. Cho CM, Hirsch R., Johnstone S. General and oral health implications of cannabis use. Australian Dental J., 2005; 50(2): 70-4.

16. Ribeiro-Araujo M., Laranjeira R., Dunn J. Cocaína: bases biológicas da administração, abstinência e tratamento. J. Bras. Psiquiatr., 1998; 47(10): 497-511.

17. Gontijo B., Bittencourt FV, Lourenço LFS. Manifestações cutâneas decorrentes do uso de drogas ilícitas. An. Bras. Dermatol., 2006; 81(4): 307-17.

18. Rosas MP, Santos CIJ, González CLG. Perforación em bóveda palatina por consumo de cocaína. Med. Oral Patol. Cir. Bucal, 2006; 11(1): 239-42.

19. Lero IMVA. Patologias e terapêuticas que interferem a prática do médico dentista, 177s. Dissertação-Universidade Fernando Pessoa, Porto, PT, 2009.

20. Ferigolo M., Medeiros FB, Barros HMT. "Êxtase": revisão farmacológica. Rev. Saúde Pública, 1998; 32(5): 487-95.

21. Cunha GH, Jorge ARC, Fonteles MMF, Sousa FCF, Viana GSB, Vasconcelos SMM. Nicotina e tabagismo. Rev. Eletr. Pesq Méd., 2007; 1(4): 45-52.
22. Borelli Neto LAA. Influência do Fumo na Doença Periodontal. J. Cient. Odont., 2011; 1(1): 3-7.

23. Maciel MED, Vargas D. Redução de danos: Uma alternativa ao fracasso no combate às drogas. Cogitare Enferm., 2015; 20(1): 207-10.

24. Tisott ZL, Hildebrandt LM, Leite MT, Martins RV, Cosentino SF. Álcool e outras drogas e a implantação da política de redução de danos no Brasil: Revisão narrativa. Rev. Bras. Ciências Saúde - USCS, 2015; 13(43): 79-10.

25. Gupta T., Shah N., Mathur VP, Dhawan A. Oral health status of a group of illicit drug users in Delhi, India. Community Dent Health, 2012; 29(1): 49-54.

26. Supera. Sistema para Detecção do Uso Abusivo e Dependência de Substâncias Psicoativas: Encaminhamento, Intervenção Breve, Reinserção Social e Acompanhamento. Módulo 1 - O uso de substâncias psicoativas no Brasil: Epidemiologia, Legislação, Políticas Públicas e Fatores Culturais. Brasília: Secretaria Nacional Antidrogas, 2006.

27. Cabral L., Mildemberg M., Assis P., Almeida P., Lindolm R., Burc MC. A ação dos anestésicos locais em usuários de cocaína. Rev. Gestão \& Saúde, 2014; 11: 22-27.

28. Falcão CAM, Ferraz MAAL. Nogueira LT. Saúde bucal em dependentes químicos. Rev. Interd. Ciênc. Saúde, 2015; 2(3): 112-121.

29. Bergamaschi CC, Monta MF, Cogo K., Franco GCN, Groppo FC, Volpato MC. Interações medicamentosas: analgésicos, anti-inflamatórios e antibióticos (Parte II). Rev. Cir. Traumatol. Buco-Maxilo-fac., 2007; 7(2): 9-18.

30. Inoue $\mathrm{G}$. et al. Cessação de tabagismo em fumantes com periodontite crônica. Braz. J. Periodontol., 2013; 23(1): 62-67.

31. Alves DM, Nai GA, Parizi JLS. Avaliação da ação do uso de drogas na saúde bucal de dependentes químicos. Colloquium Vitae, 2013; 5(1): 40-58.

Submetido em: 27-9-2015

Aceito em: 18-4-2016 